\title{
MIMO SAR FORMATIONS: ORBITAL DIAMETER AND SYNCHRONIZATION TOLERANCES
}

\author{
Andrea Monti Guarnieri ${ }^{1}$, Davide Giudici ${ }^{2}$, Pietro Guccione ${ }^{3}$, Marco Manzoni ${ }^{1}$, Fabio Rocca ${ }^{1}$ \\ ${ }^{1}$ Dipartimento di Elettronica, Informazione e Bioingegneria, Politecnico di Milano, Pzza Leonardo da Vinci, 32, 20133 Milano, Italy \\ ${ }^{2}$ Aresys srl, via Flumendosa 16, 20132 Milano, Italy \\ ${ }^{3}$ Dipartimento di Ingegneria Elettrica e dell'Informazione, Politecnico di Bari, Via E. Orabona, 4, 70126 Bari, Italy
}

KEY WORDS: SAR, MIMO Radar, Interferometry, Tomography, Radar imaging.

\begin{abstract}
:
Multiple-Input-Multiple Output (MIMO) Synthetic Aperture Radar (SAR) along-track formations can be used to fraction the power resources into compact, lightweight and cost-effective satellites, or to extend the swath coverage beyond the limit provided by a small antenna. In this second case, the Pulse Repetition Frequency (PRF) is kept low by implementing an inversion that solves up to N-1 ambiguities, given $\mathrm{N}$ observations. The simultaneous illumination - that allows for the $\mathrm{N}^{2}$ gain due to the coherent combination of the $\mathrm{N}$ transmitters and the $\mathrm{N}$ receivers, is analyzed and shown not to be critical, as the more than $\mathrm{N}=2$ sensors are assumed. Performance is evaluated for the $\mathrm{N}=2$ and $\mathrm{N}=3$ cases and compared with the Single Input Multiple Output formations, where one sensor is transmitting, and all are receiving. Finally, the impact of the across-track deviation from the orbit is modeled and evaluated.
\end{abstract}

\section{INTRODUCTION}

Loose formations of mini SAR satellites have been studied in the SIMO case (Aguttes, 2003; Cheng et al., 2017; Gebert et al., 2010; Guccione et al., 2020; Mittermayer et al., 2016). Such SIMO formation allows multi-sensor coherent imaging achieving a power gain proportional to the number of $\mathrm{N}$ sensors, thanks to the fractioning of the antenna while keeping the advantages in terms of flexibility, scalability, and robustness of a constellation. Furthermore, using $\mathrm{N}$ satellites along-track, the PRF can be reduced $\mathrm{N}_{\mathrm{r}}$ times to expand the swath. Then, $\mathrm{N}_{\mathrm{r}} \leq \mathrm{N}$ ambiguities can be mitigated using an adaptive PRF and twin antennas, while keeping the formation loose but much shorter than the antennas footprint.

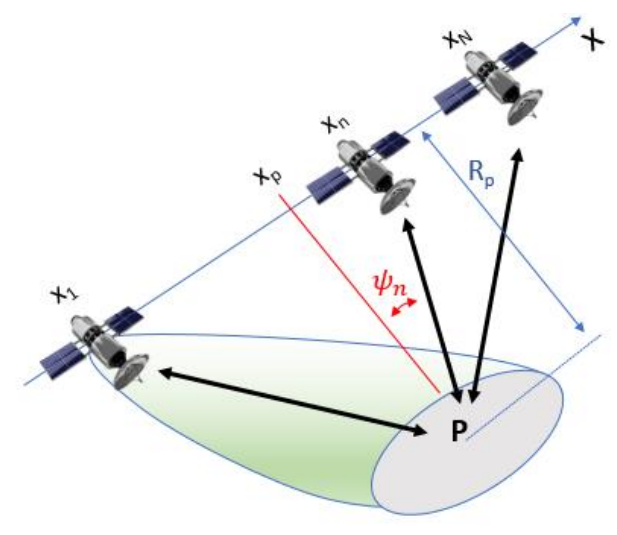

Figure 1. Geometry of a MIMO-N formation.

Here we address a MIMO case (Giudici, 2019), an evolution of the concept where $\mathrm{N}$ satellites transmit simultaneously the same code at the same frequency bands, the geometry is shown in Figure 1 for the MIMO-3 configuration.

The resultant from the combination of the $\mathrm{N}$ transmissions with random phases causes fluctuations in the illumination of a target, that change within the synthetic aperture. The total contribution to the target $\mathrm{P}$ is the coherent combination of the signals from the $\mathrm{N}$ active satellites:

$$
\begin{aligned}
& s=\sum_{n=1}^{N} s_{0} \exp \left(j \frac{2 \pi}{\lambda} \frac{x_{n}-x_{p}}{\cos \psi_{n}}\right) \\
& =s_{0} \exp \left(j \frac{2 \pi x_{p}}{\lambda}\right) \sum_{n=1}^{N} \exp \left(j \frac{2 \pi}{\lambda} \frac{x_{n}}{\cos \psi_{n}}\right)
\end{aligned}
$$

where $\quad s_{0}=$ signal amplitude, assumed identical for all sensors $\mathrm{x}_{\mathrm{p}}, \mathrm{x}_{\mathrm{n}}=$ along track coordinates of target and satellites $\lambda=$ wavelength $\psi_{\mathrm{n}}=$ squint angle of the $\mathrm{n}$-th satellite

The summation in (1) involves random phases that, for each target, change with the position of the sensors:

$$
\phi_{n}=\frac{2 \pi}{\lambda} \frac{x_{n}}{\cos \psi_{n}} \simeq \frac{2 \pi}{\lambda} R_{p}-\frac{2 \pi}{\lambda} \frac{x_{n}^{2}}{2 R_{p}}
$$

This term changes many tens of cycles over the synthetic aperture, which is $\lambda R_{\mathrm{p}} / \mathrm{L}_{\mathrm{a}}$ for a typical LEO mission, like Sentinel-1, where $R_{\mathrm{p}}=850 \mathrm{~km}, \lambda=5.6 \mathrm{~cm}$, and the antenna length is $\mathrm{L}_{\mathrm{a}}=12 \mathrm{~m}$. Therefore, the instantaneous power on each target $\mathrm{P}$ is the sum of random phasors, proportional to:

$$
P=\left|\sum_{n} \exp \left(j \phi_{n}\right)\right|^{2}
$$

The power $\mathrm{P}$ changes randomly with the along-track positions of the sensors, $\mathrm{x}_{\mathrm{n}}$, resulting in a fluctuation of the target amplitude along the synthetic aperture. These fluctuations can be compensated by a proper inversely weighted average of the $\mathrm{N}$ received signal, that we discuss in the next section, provided that the amplitude is high enough to counteract for thermal noise.

To evaluate such occurrence, we have plotted in Figure 2 the Cumulative Distribution Function (CDF) of the random variable $P$ in (3), that is the probability that the power exceeds a certain threshold, for the cases $\mathrm{N}=2 \ldots 5$. The $\mathrm{CDF}$ has been normalized to the mean power: 


$$
E[P]=E\left[\sum_{n, m} \exp \left(j\left(\phi_{n}-\phi_{m}\right)\right)\right]=N
$$

The behaviour of the distribution for small values of $\mathrm{P}$ - the most critical, is evidenced in the zoom on the right of the figure. This shows a worst-case for $\mathrm{N}=2$, with the highest probability of very small values, a best one, for $\mathrm{N}=3$, whereas for large $\mathrm{N}$, the normalized power converges to an exponential distribution whose $\mathrm{CDF}$ is:

$$
p\left(\frac{P}{N} \geq x\right)=1-\exp (x) \text { for } x \geq 0
$$
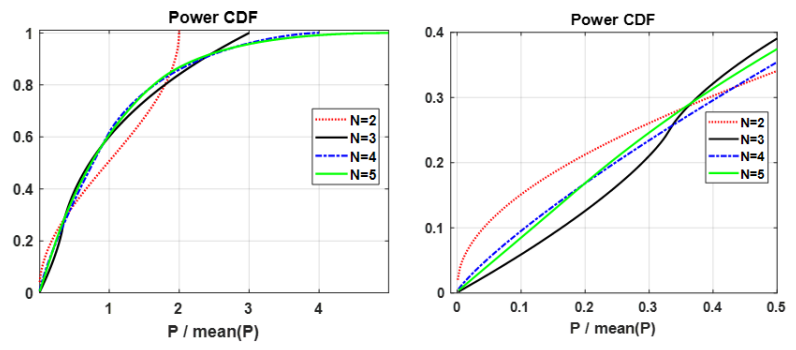

Figure 2. Cumulative Probability Distribution of power of the sum of random phasors, normalized to the mean.

\section{HIGH-RESOLUTION MIMO IMAGING}

The time-variable illumination causes unwanted distortion in the image. These distortions can be studied by considering that SAR acquisition is linear, and then approximating the Impulse Response Function (IRF) of the signal transmitted by the $m$-th sensor and received by the $n$-th one, by a pure monodimensional phase term (Gebert et al., 2009; Richard Bamler, 1992):

$$
\begin{gathered}
h_{n m}(x)=A\left(x-x_{0}\right) \cdot \exp \left(j \frac{2 \pi}{\lambda} R_{n m}(x)\right) \\
R_{n m}(x)=\sqrt{\left(x-x_{n}\right)^{2}+R_{p}^{2}}+\sqrt{\left(x-x_{m}\right)^{2}+R_{p}^{2}}
\end{gathered}
$$

where $A\left(x-x_{0}\right)$ is the antenna pattern, that we assume identical for all satellites and centered in the same position $\mathrm{x}_{0}$

$R_{n m}$ is the overall path from the $m$-th transmitter to the target (assumed in $\mathrm{x}=0$ ) and the $\mathrm{n}$-th receiver.

The bistatic path $\mathrm{R}_{\mathrm{nm}}$ can be approximated for $\mathrm{R}_{\mathrm{p}}$ large by linearizing (6):

$$
\begin{gathered}
R_{n m}(x) \approx 2 R_{p}+\frac{\left(x-x_{n m}\right)^{2}}{2 R_{p}}+\frac{x_{n m}}{2 R_{p}} \\
x_{n m}=0.5 \cdot\left(x_{n}+x_{m}\right)
\end{gathered}
$$

The IRF from the target to the generic receiver, the $n$-th observation, can be derived by summing the $\mathrm{N}$ transmissions:

$$
h_{n}(x)=\sum_{m} \exp \left(j \frac{2 \pi}{\lambda} \frac{\left(x-x_{n m}\right)^{2}}{2 R_{p}}\right)
$$

where we have ignored - for simplicity - a phase offset that depends on $\mathrm{n}, \mathrm{m}$ and we assumed constant antenna pattern. The summation in (8) is responsible for the amplitude fluctuations, discussed after (1). However such fluctuation can be mitigated by combining the $\mathrm{N}$ different observations of the same target modeled by (8), for $n=1 \ldots N$. The simplest approach is to handle the reconstruction in the frequency domain since the convolution by the IRF becomes a frequency-varying weight. The Fourier Transform of (8) is:

$$
H_{n}\left(f_{x}\right)=\sum_{m} \exp \left(-j \pi R_{p} \frac{f_{x}^{2}}{f_{0}}\right) \exp \left(-j 2 \pi x_{n m} f_{x}\right)
$$

Where $f_{0}$ is the carrier frequency. The Fourier Transform of each observation, $\mathrm{Y}_{\mathrm{n}}\left(\mathrm{f}_{\mathrm{x}}\right)$, under the simplifications here introduced is the summation of the spectrum of the scene $D\left(f_{x}\right)$ within all the bistatic channels:

$$
Y_{n}\left(f_{x}\right)=\mathrm{H}_{0}\left(\mathrm{f}_{\mathrm{x}}\right) \sum_{m} H_{1, n m}\left(f_{x}\right) \cdot D\left(f_{x}\right)
$$

where $\mathrm{H}_{0}$, the first in (9), is responsible for the quadratic phase $\mathrm{H}_{1, \mathrm{~nm}}$ is the second term in (9) that accounts for the alongtrack shift of the equivalent monostatic phase center, $\mathrm{x}_{\mathrm{nm}}$.

The forward model of the observed SAR spectrum (10) can be written in matrix format:

$$
\mathbf{Y}\left(f_{x}\right)=\mathrm{H}_{0}\left(\mathrm{f}_{\mathrm{x}}\right) \cdot \mathbf{H}_{1}\left(f_{x}\right) \cdot D\left(f_{x}\right)
$$

where $\quad \mathbf{Y}$ is the $[\mathrm{N}, 1]$ column vector with the observations $Y_{n}$ $\mathbf{H}_{1}$ the [N,1] column vector with the shifts $\mathrm{H}_{1, \mathrm{~nm}}$ and inverted in LS to derive the best guess for the SAR reflectivity:

$$
\widehat{\mathrm{D}}=\mathrm{H}_{0}^{-1} \cdot\left(\mathbf{H}_{1}^{*} \cdot \mathbf{H}_{1}\right)^{-1} \cdot \mathbf{H}_{1}^{*} \cdot \mathbf{Y}
$$

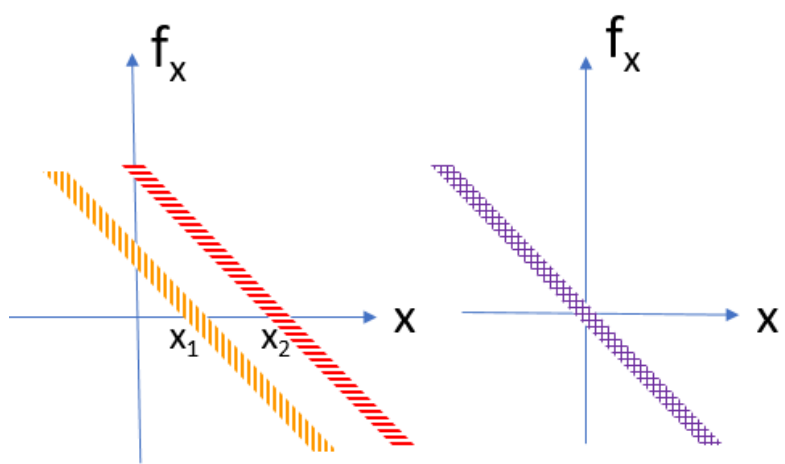

Figure 3. Interpretation of the MIMO-2 reconstruction in the time frequency diagram, representing: on the left, the contribution of a target in $\mathrm{x}=0$, observed by the $\mathrm{N}=2$ sensors, located in $\mathrm{x}_{1}, \mathrm{x}_{2}$ and on the right the MIMO recombination.

The MIMO recombination can be expressed as follows:

$$
\widehat{D}=\mathrm{H}_{0}^{-1} \frac{\sum_{n}\left(\sum_{m} \exp \left(-j 2 \pi x_{n m} f_{x}\right)\right) \cdot Y_{n}^{*}}{\sum_{n}\left|\sum_{m} \exp \left(-j 2 \pi x_{n m} f_{x}\right)\right|^{2}}
$$

The interpretation of the MIMO reconstruction is given in Figure 3 for the case $\mathrm{N}=2$. On the left, one finds the Doppler phase history of the same target, as seen by the $\mathrm{N}=2$ sensors. The amplitudes, for each frequency, are different and random due to (3). For each frequency, the along-track contribution is also shifted: for example, the zero Doppler for the two sensors are respectively at $\mathrm{x}_{1}$ and $\mathrm{x}_{2}$. However, the MIMO recombination in (12), (13) performs a summation - with a proper spectral equalization (in the denominator) and time-domain shift aimed to reconstruct a unique Doppler phase that will then be focused by the inverse operator $\mathrm{H}_{0}^{-1}$.

In the real world, one has to compensate further for the phase terms in (7), and for the range migration and secondary range migration, as discussed in (Cheng et al., 2017; Gebert et al., 2009; Guccione et al., 2020; Mittermayer et al., 2016) for the SIMO cases. 


\subsection{Power gain}

The role of an additional noise can be evaluated by applying the inversion (12) to the forward model (11), corrected with additional noise:

$$
\begin{gathered}
\widehat{\mathrm{D}}=\mathrm{H}_{0}^{-1} \cdot\left(\mathbf{H}_{1}^{*} \cdot \mathbf{H}_{1}\right)^{-1} \cdot \mathbf{H}_{1}^{*} \cdot\left(\mathrm{H}_{0} \cdot \mathbf{H}_{1} \cdot D+W\right) \\
=D+W
\end{gathered}
$$

where $\mathrm{D}$ and $\mathrm{W}$ are respectively the SAR signal and the noise. The estimated reflectivity is then unbiased, but the noise adds a variance that can be computed from (12):

$$
\begin{gathered}
\sigma_{D}^{2}=E\left[\mathrm{H}_{0}^{-1}\left(\mathbf{H}_{1}^{*} \mathbf{H}_{1}\right)^{-1} \mathbf{H}_{1}^{*} \mathbf{H}_{1}\left(\mathbf{H}_{1}^{*} \mathbf{H}_{1}\right)^{-1} \mathrm{H}_{0}^{-1 *}\right] \sigma_{w}^{2} \\
=\left(\mathbf{H}_{1}^{*} \mathbf{H}_{1}\right)^{-1} \sigma_{w}^{2}
\end{gathered}
$$

We define the Recombination Gain (RG) as:

$$
R G=\frac{\sigma_{D}^{2}}{\sigma_{w}^{2}}=\frac{1}{\left(\mathbf{H}_{1}^{*} \mathbf{H}_{1}\right)^{-1}}
$$

That is to be evaluated as a figure of quality, together with the impulse response function. Notice that by inserting (8) into (16) we get that the mean value of the $\mathrm{RG}$ is:

$$
\begin{aligned}
& \mathrm{E}[R G]=E\left[\frac{1}{\left(\mathbf{H}_{1}^{*} \mathbf{H}_{1}\right)^{-1}}\right]=E\left[\mathbf{H}_{1}^{*} \mathbf{H}_{1}\right]=E\left[\sum_{n}\left|h_{n}\right|^{2}\right]= \\
& E\left[\sum_{n}\left|\sum_{m} \exp \left(j \frac{2 \pi}{\lambda} \frac{\left(x-x_{n m}\right)^{2}}{2 R_{p}}\right)\right|^{2}\right]=N^{2}
\end{aligned}
$$

that accounts for the gain due to fractioning the power and antenna resources into the $\mathrm{N}$ transmitters and receivers.

\subsection{Examples from simulations}

To appreciate the capabilities of reconstructing the full resolution reflectivity, we have performed a full 2D (range, azimuth) simulation and focusing of the two cases of interest: the MIMO2 and the MIMO- 3 combinations. The selection of the two cases was driven by the fact that represents the worst and best cases.

\begin{tabular}{|l|l|l|}
\hline Parameter & MIMO-2 & MIMO-3 \\
\hline Orbit altitude & \multicolumn{2}{|c|}{$500 \mathrm{~km}$} \\
\hline Sensors velocity & \multicolumn{2}{|c|}{$7.6 \mathrm{~km} / \mathrm{s}$} \\
\hline Incidence angle & \multicolumn{2}{|c|}{$29^{\circ}$} \\
\hline Frequency & \multicolumn{2}{|c|}{$5.4 \mathrm{GHz}$} \\
\hline Bandwidth & \multicolumn{2}{|c|}{$52 \mathrm{MHz}$} \\
\hline SNR (peak to average) & \multicolumn{2}{|c|}{$13 \mathrm{~dB}$} \\
\hline Antenna Length & $6 \mathrm{~m}$ & $4 \mathrm{~m}$ \\
\hline Antenna bandwidth & $2530 \mathrm{~Hz}$ & $3800 \mathrm{~Hz}$ \\
\hline PRF & $2940 \mathrm{~Hz}$ & $3810 \mathrm{~Hz}$ \\
\hline Azimuth resolution & $3.5 \mathrm{~m}$ & $2.9 \mathrm{~m}$ \\
\hline
\end{tabular}

Table 1. Parameters of the MIMO formation tested

The parameters for the simulation are listed in Table 1. The aim was to resemble Sentinel-1 geometry, but the orbit height has been reduced and, consequently, the synthetic aperture, to speed up the numerical simulations. In the simulations, an additional noise has been introduced to represent a realistic case.

The IRF before and after the MIMO reconstruction, together with its spectrum, are represented in Figure 4 and Figure 5 respectively for the cases of MIMO-2 and MIMO-3. The simulations were fully $2 \mathrm{D}$, yet only the azimuth cut of the IRF is shown, as in the range direction no distortion is found.

One can notice that the reconstruction of the MIMO-3 is far better, due to the presence of less spectral notches. This is due to the high occurrence, in the MIMO-2 configuration, of the two transmitted signals to combine with the opposite phase, that is also clear in Figure 2. To mitigate this effect one could transmit a different power between the two systems. An example of a MIMO-2 reconstruction with $30 \%-70 \%$ power unbalance is shown in Figure 6. The frequency-domain nulls are less evident, showing the effectiveness of the approach. However, one has to account for a reduced fractioned MIMO gain.

A full evaluation of performance is reported in Table 2. The results are in the range expected and provide evidence of a good SAR system. The MIMO-3 has little worse performance in terms of azimuth sidelobes, which is due to the lower oversampling, the ratio between the PRF and the antenna bandwidth, in Table 1.
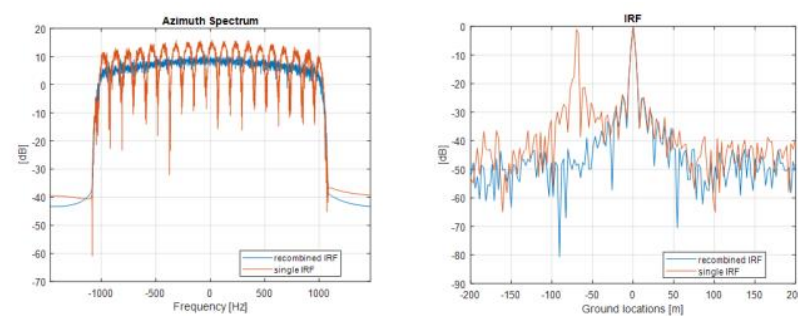

Figure 4. Simulation of the IRF of a MIMO-2 recombination in presence of noise. Left: in the spectral domain, right: in the time domain. Observations are in red, reconstruction in blue.
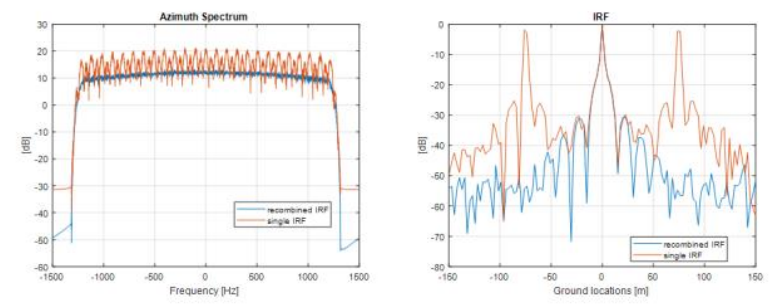

Figure 5. Simulation of the IRF of a MIMO-3 recombination.
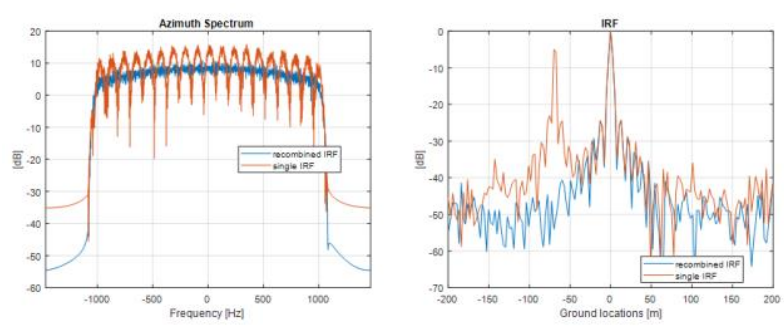

Figure 6. Simulation of the IRF of a MIMO-2 recombination with $30 \%-70 \%$ power unbalance between the two sensors.

\begin{tabular}{|l|c|c|}
\hline Performance parameters & MIMO-2 & MIMO-3 \\
\hline Recombination Gain & 4.0 & 9.0 \\
\hline SNR formation [dB] & 19.0 & 22.5 \\
\hline Peak height (1x1 res cell) & 1.5 & 3 \\
\hline Azimuth resolution [m] & 3.4 & 2.7 \\
\hline Slant range resolution [m] & 2.2 & 2.2 \\
\hline Az PSLR [dB] & -16.8 & -15.5 \\
\hline Rg PSLR [dB] & -13.4 & -13.4 \\
\hline Az ISLR (10 res cells) [dB] & -13.8 & -12.5 \\
\hline Rg ISLR (10 res cells) [dB] & -10.7 & -10.7 \\
\hline
\end{tabular}

Table 2. Performance of the MIMO reconstruction

\section{WIDE SWATH HIGH RESOLUTION}

The availability of $\mathrm{N}$ observations for each target spectral contribute can be efficiently exploited to solve for $\mathrm{N}$ ambiguities, similarly to the SIMO case, (Aguttes, 2003; Cheng et al., 2017; Gebert et al., 2010; Guccione et al., 2020; Mittermayer et al., 2016). The idea is shown in the time-frequency diagram of Figure 7 , that draws the instantaneous contribution of one target, located in $\mathrm{x}_{\mathrm{p}}$, and other two, located in $\mathrm{x}_{\mathrm{a} 1}$ and $\mathrm{x}_{\mathrm{a} 2}$ whose spectrum is 
folded by the $\mathrm{N}$ undersampling. This occurs since the PRF is kept $\mathrm{N}$ times lower that the antenna bandwidth, shaded in grey.

Here, the $\mathrm{N}$ observations allow to reconstruct the full spectrum by solving the $\mathrm{N}$ unknowns, and while the low PRF allows extending the coverage to multiple swaths (Gebert et al., 2010; Krieger et al., 2004).

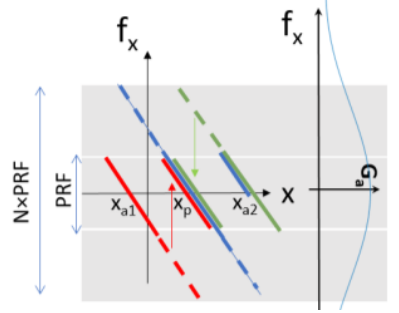

Figure 7. The MIMO-N (N=3 here) allows to solve for the $\mathrm{N}-1$ ambiguous targets, placed at $\mathrm{x}_{\mathrm{a} 1}$ and $\mathrm{x}_{\mathrm{a} 2}$ in the time-frequency diagram, whose spectra overlap with the target in $\mathrm{x}_{\mathrm{p}}$.

The formulation of the forward model is an extension of the one in (11):

$$
\mathbf{Y}\left(f_{x}\right)=\mathrm{H}_{0}\left(\mathrm{f}_{\mathbf{x}}\right) \cdot \mathbf{H}_{1}\left(f_{x}\right) \cdot \mathbf{D}\left(f_{x}\right)
$$

where:

1) D is the $\left[\mathrm{N}_{\mathrm{r}}, 1\right]$ column vector with the scene spectral samples of the scene taken at multiple of the PRF:

D

$$
=\left[\begin{array}{lll}
D\left(f_{x}+0 \cdot \frac{P R F}{v}\right) & \ldots & D\left(f_{x}+\left(N_{r}-1\right) \cdot \frac{P R F}{v}\right)
\end{array}\right]^{T}
$$

2) $\mathbf{H}_{1}[\mathrm{~N}, 1]$ is a $\left[\mathrm{N}, \mathrm{N}_{\mathrm{r}}\right]$ matrix whose generic element is:

$$
\begin{aligned}
& H_{n, r}\left(f_{x}\right)=\sum_{m} \exp \left(-j 2 \pi \lambda R_{p}\left(f_{x}+r\right.\right. \\
&\left.\left.\cdot \frac{P R F}{v}\right)\right) \exp \left(-j 2 \pi x_{n m}\left(f_{x}+r \cdot \frac{P R F}{v}\right)\right)
\end{aligned}
$$

The inversion is formally identical to (12) but involves matrixes in place of vectors. Therefore, in the estimation of the SNR in (17) the total noise power involves the evaluation of the trace:

$$
S N R=\frac{\sigma_{D}^{2}}{\sigma_{w}^{2}}=\frac{1}{\operatorname{Tr}\left(\left(\mathbf{H}_{1}^{*} \mathbf{H}_{1}\right)^{-1}\right)}
$$

Finally, we remark that a full MIMO should account also for the phase terms that have been dropped in (8), and then implement the proper range migration correction, as detailed in the SIMO processing in (Guccione et al., 2020).

\subsection{Performance comparison with the SIMO case}

The MIMO formation with ambiguity cancellation is here compared with the corresponding SIMO, where only one sensor transmits, and all the others receive.

As a MIMO-N can solve up to N-1 ambiguities, that are however symmetric, as shown in Figure 7, the interest is here for odd formations. In Figure 7 we compare the result of MIMO-3 recombination with the corresponding SIMO-3. The parameters are the same assumed in Table 1, but for the PRF that has been reduced to $1270 \mathrm{~Hz}$ to allow for scanning three swaths, getting a coverage quite similar to Sentinel-1 in IW mode.

One can observe from the figure that the IRF is quite good, and also the spectra, where the fluctuations are due to the contribution of added noise. The evaluation of performance, in Table 3, show minimal differences between the two cases, apart from the SNR gain computed according to (19), which is practically $\mathrm{N}=3$ for the SIMO and $\mathrm{N}^{2}=9$ for the MIMO.
In performing the simulations we have placed the sensors in the optimal along-track positions. For the SIMO these are:

$$
x_{n}=\frac{v}{P R F} \cdot\left(k+\frac{n}{N}\right)
$$

where $\mathrm{k}$ is an integer. For the MIMO case, the locations have been found numerically.
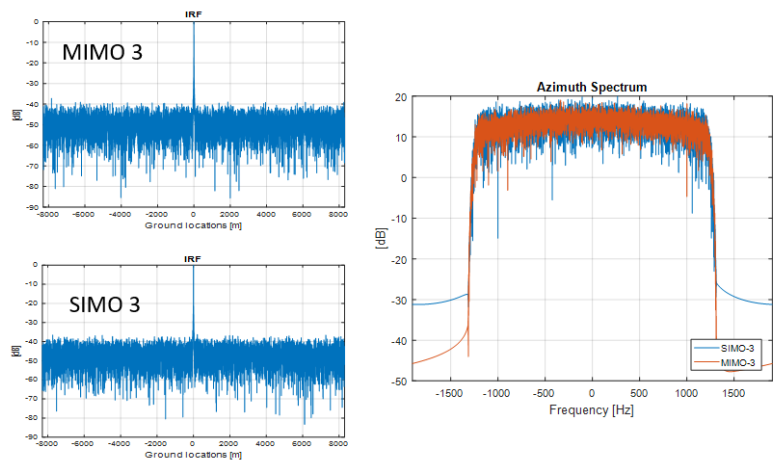

Figure 7. The MIMO-N ( $\mathrm{N}=3$ here) allows to solve for the $\mathrm{N}-1$ ambiguous targets, placed at $\mathrm{x}_{\mathrm{a} 1}$ and $\mathrm{x}_{\mathrm{a} 2}$ in the time-frequency diagram, whose spectra overlap with the target in $\mathrm{x}_{\mathrm{p}}$.

\begin{tabular}{|l|l|l|}
\hline Performance parameter & SIMO-3 & MIMO-3 \\
\hline Recombination Gain & 3.0 & 8.97 \\
\hline SNR formation [dB] & 17.7 & 22.5 \\
\hline SNR monostatic [dB] & 13.0 & 13.0 \\
\hline Peak height (1x1 res cell) & 3 & 3 \\
\hline $\begin{array}{l}\text { Point to target ambiguity ratio } \\
\text { [dB] }\end{array}$ & 29.4 & 32.2 \\
\hline Azimuth resolution [m] & 2.7 & 2.7 \\
\hline $\begin{array}{l}\text { Peak to Sidelobe Ratio - azimuth } \\
\text { [dB] }\end{array}$ & -15.5 & -15.3 \\
\hline
\end{tabular}

Table 3. Performance of the MIMO reconstruction

To evaluate the sensitivity respect to the relative positioning of the sensors, a Monte Carlo analysis has been run to evaluate the average Recombination Gain (RG) and PTAR (including only the first ambiguities or all) as a function of the fine positioning of the sensors. The mean values are reported in Figure 8: notice that the sensitivity is small respect to the reconstructed image sampling, $v /(N \cdot P R F)$, that is in the order of a couple of meters.
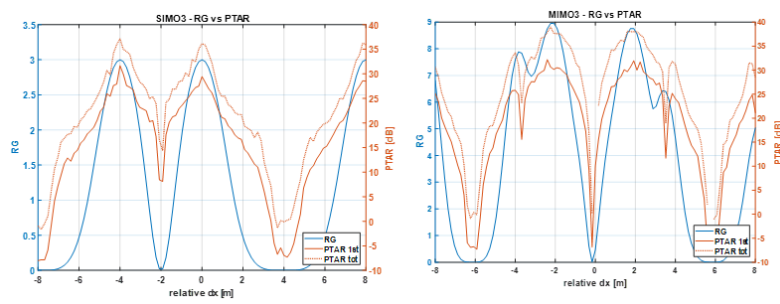

Figure 8. Evaluation of the average Recombination Gain (RG) and of the PTAR (by assuming just one or all the ambiguities) as function of the fine positioning of the sensors, for the cases of a formation SIMO-3, left, and the MIMO-3, right.

\subsection{Impact of the Baseline}

So far, we assumed the sensors in the formation to follow one another on the same rectilinear orbit. Errors in the across-track positioning introduce additional phases that are dependent on the precise position of the target, which differs from the ambiguities. This makes the compensation of the consequent error quite difficult or even impossible. The case has already been 
approached in the SIMO case, (Guccione et al., 2020). The reference geometry is shown in Figure 9, the across-track, range space. In the figure, $\mathrm{B}_{\mathrm{n}}$ the baseline error of the generic sensor, respect to a reference master, that, for the SIMO case, is assumed to be the transmitter. The figure shows that two targets at approximately the same distance from the satellite, contribute with am interferometric phase shift (Rosen et al., 2000):

$$
\phi_{n m}=\frac{2 \pi q}{R_{P} \lambda \sin \theta}\left(B_{n}-B_{m}\right)
$$

where $\theta=$ local in incidence angle $\mathrm{q}=$ target elevation

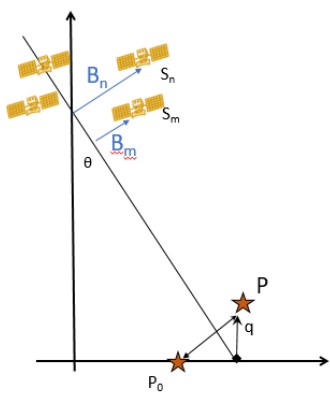

Figure 9. Sketch of the geometries for the analysis of the impact of the normal baselines

The difference between the SIMO and the MIMO acquisition is implied in the forward matrix $\mathbf{H}_{1}$ in (18), whose elements, in the SIMO case are the single bistatic phase from the sole transmitter to the generic receiver, whereas for the MIMO is the sum extended to all the $\mathrm{N}$ transmitters. The resulting error has been computed under the assumption of small baselines in (Guccione et al., 2020) in the SIMO case. In the MIMO case the uncompensated ambiguity leads to an error is:

$$
y=d_{a} \sum_{n, m=1 ; n \neq m}^{N} \exp \left(\frac{2 \pi}{R_{P} \lambda \sin \theta}\left(B_{n}-B_{m}\right)\right)
$$

where $\quad d_{a}=$ complex reflectivity of the ambiguity

$$
\mathrm{y}=\text { residual ambiguity error }
$$

$\mathrm{x}_{\mathrm{p}}, \mathrm{x}_{\mathrm{n}}=$ along-track coordinates of target and satellites $\lambda=$ wavelength

$\psi_{\mathrm{n}}=$ squint angle of the $\mathrm{n}$-th satellite

One point is that the error is always real since the summation in (22) involves even terms. Furthermore, this is responsible for a coherence:

$$
\gamma_{h}=\frac{E\left[\left|\sum_{n, m=1 ; n \neq m}^{N} \exp \left(\frac{2 \pi q}{R_{P} \lambda \sin \theta}\left(B_{n}-B_{m}\right)\right)\right|^{2}\right]}{N^{2}}
$$

that, assuming a zero mean, normal distribution for both the elevations and the baselines can be linearly approximated to

$$
\gamma_{h} \simeq 1-\frac{N-1}{N}\left(\frac{2 \pi}{R_{P} \lambda \sin \theta}\right)^{2} \frac{\sigma_{q}^{2} \sigma_{b}^{2}}{2}
$$

where $\sigma_{q}^{2}$ and $\sigma_{b}^{2}$ are the standard deviation of the elevation and baseline respectively.

The result in (24) compares with the SIMO case but assuming a double value for the baseline standard deviation. As a practical example, in the MIMO-3 case, by assuming the parameters in Table 1 , and $\sigma_{\mathrm{q}}=100 \mathrm{~m}$, we would need a baseline dispersion of about $10 \mathrm{~m}$ to keep the coherence higher than 0.9. This will then result in $\sigma_{b}=12 \mathrm{~m}$ if we assume the Sentinel-1 case with orbit height of $700 \mathrm{~km}$ in place of 500 .

\section{CONCLUSIONS}

The simultaneous MIMO formation here presented provides a valuable generalization of the well-known SIMO concept. The SNR gain of $\mathrm{N}^{2}$ has been confirmed even in the worst case of $\mathrm{N}=2$, attaining a good IRF. If the formation is exploited to enhance the coverage, by reducing the PRF, performance in terms of signal to ambiguity ratio and sidelobes in the impulse response function are shown to be comparable with the SIMO cases. Also, the along-track positioning has proven a similar sensitivity, where accuracies better than a fraction $L_{a} / N$ are required. Such along-track metric accuracy can be well achieved by exploiting the split antenna, proposed in (Guidici et al, 2019). The sensitivity respect to the normal baselines is twice respected to the SIMO, however, the errors will compensate giving always phase preserving images.

\section{REFERENCES}

Aguttes, J.P., 2003: The SAR train concept: required antenna area distributed over $\mathrm{N}$ smaller satellites, increase of performance by N, in IGARSS 2003 , pp. 542-544 vol.1. doi.org/10.1109/IGARSS.2003.1293836

Cheng, P., Wan, J., Xin, Q., Wang, Z., He, M., Nian, Y., 2017: An Improved Azimuth Reconstruction Method for Multichannel SAR Using Vandermonde Matrix, IEEE Geoscience and Remote Sensing Letters 14, 67-71

doi.org/10.1109/LGRS.2016.2626309

Gebert, N., Krieger, G., Moreira, A., 2010: Multichannel Azimuth Processing in ScanSAR and TOPS Mode Operation. IEEE Transactions on Geoscience and Remote Sensing 48, 2994-3008. doi.org/10.1109/TGRS.2010.2041356

Gebert, N., Krieger, G., Moreira, A., 2009. Digital Beamforming on Receive: Techniques and Optimization Strategies for HighResolution Wide-Swath SAR Imaging. IEEE Transactions on Aerospace and Electronic Systems 45, 564-592,

Giudici, D., 2019. Synthetic Aperture Radar Detection System, Patent Request 102019000021 294, 2019.

Guccione, P., Monti Guarnieri, A., Rocca, F., Giudici, D., Gebert, N., 2020. Along-Track Multistatic Synthetic Aperture Radar Formations of Minisatellites. Remote Sensing 12. https://doi.org/10.3390/rs12010124

Krieger, G., Gebert, N., Moreira, A., 2004. Unambiguous SAR signal reconstruction from nonuniform displaced phase center sampling. IEEE Geoscience and Remote Sensing Letters 1, 260 264. https://doi.org/10.1109/LGRS.2004.832700

Mittermayer, J., Lopez-Dekker, P., Kraus, T., Krieger, G., 2016. Small Satellite Dispersed SAR - an Exemplary Configuration, in Proceedings of EUSAR 2016: 11th European Conference on Synthetic Aperture Radar. pp. 1-4.

Richard Bamler, 1992. A comparison of range-Doppler and wave-number domain SAR focusing algorithms. IEEE Transactions on Geoscience and Remote Sensing 30, 706-713.

Rosen, P., Hensley, S., Joughin, I.R., Li, F.K., Madsen, S., Rodriguez, E., Goldstein, R.M., 2000. Synthetic Aperture Radar Interferometry. Proceedings of the IEEE 88, 333-382. doi.org/10.1109/5.838084 